\title{
HAEMATOGENOUS OSTEITIS OF INFANCY
}

\author{
BY \\ WALLACE M. DENNISON and DUNCAN A. MACPHERSON \\ From the Royal Hospital for Sick Children, Glasgow
}

(RECEIVED FOR PUBLICATION JANUARY 15, 1952)

\begin{abstract}
During the past 30 years there has been much contradictory literature on haematogenous osteitis in infancy (Thomson and Lewis, 1950). In infancy the disease differs in so many respects from osteitis in older children that a separate consideration of haematogenous osteitis in children under 2 years of age is desirable. Even in this limited age group we must consider the disease under two headings: (1) streptococcal osteitis of infancy and (2) osteitis of the newborn.
\end{abstract}

\section{Streptococcal Osteitis of Infancy}

The differences between streptococcal osteitis of infancy and acute osteitis in older children have been emphasized by many authors, notably Paschlau (1933), Green (1935), White (1937), Compere, Schnute and Cattell (1945) and Blanche (1952). In this form of osteitis severe toxaemia is unusual and abscess formation and joint infections are common-probably due to the thin and porous infantile cortex and delicate epiphyseal cartilage. Sequestrum formation is rare and any sequestra are usually absorbed spontaneously. Bone deformities are quickly rectified by growth and full joint function is restored early. Even before the introduction of antibiotics, these cases responded well to minor surgical intervention (Dennison, 1948). In the communication from which this statement is taken streptococcal infection of bone is described under the heading of 'subacute osteitis'. The prime consideration in these cases is treatment of the systemic upset and not the local bone lesion. Early drainage does not decrease the amount of bone destruction nor does it limit systemic manifestations. Although these infections have been classed as subacute, poorly-timed surgical intervention may jeopardize the child's life.

Since the introduction of penicillin therapy in bone infections during 1945, 19 cases of streptococcal osteitis of infancy have been treated in one unit of the Royal Hospital for Sick Children, Glasgow. All made a complete and uneventful recovery.

\section{Osteitis of the Newborn}

Although it is not a common condition, osteitis of the newborn is well known. Fraser (1924) stated that the disease was almost always fatal, whereas Dillehunt (1935), Cass (1940) and Stone (1942) regarded the disease as benign and all three gave a good prognosis for life and function. The confusion has been largely clarified by Greengard (1946) and Thomson and Lewis (1950). These authors pointed out that there were two forms of the disease, one benign and one severe.

Since 1945, 109 cases of acute haematogenous osteitis have been treated in one unit of the Royal Hospital for Sick Children, Glasgow. In all but two cases the infecting organism was a coagulasepositive staphylococcus. (Infections due to the pneumococcus and streptococcus each occurred once.) In this series seven patients were in the neonatal age group and in this group all infections were staphylococcal. During this period many examples of subacute osteitis (Dennison, 1948) were treated in the unit. Among these were 12 examples of the benign form of neonatal osteitis. In this group the infecting organism was a coagulasepositive staphylococcus in 10 cases and a haemolytic streptococcus in two.

In its benign form neonatal osteitis affects only one bone, there is little systemic upset, and even before the introduction of chemotherapy and antibiotics the prognosis was good. In the present series of staphylococcal infections the disease affected the maxilla (four cases), clavicle (one case), humerus (two cases), femur (two cases) and calcaneus (one case). In all cases recovery was complete and without deformity although the staphylococcus was resistant to penicillin in five cases.

The severe form of neonatal osteitis is also known as generalized osteitis of the newborn. The infection is systemic and several bones may be involved with multiple swellings of the extremities (Athanassiadis, 1951). The diagnosis is confirmed by blood culture and by radiographic changes. There is a marked tendency to early infection of 


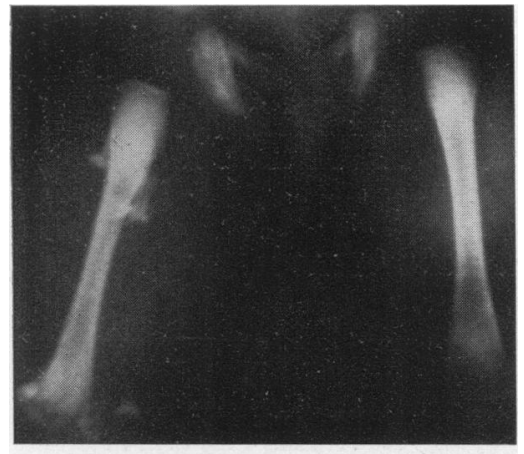

FIG. 1

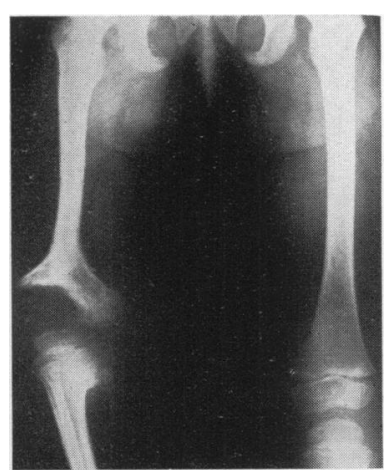

FIG. 2
FIG. 1.-Radiographic appearances four weeks after the onset of neonatal staphylococcal osteitis. Subperiosteal new bone is seen in the upper third of shaft and also severe damage to the lower metaphysis and epiphysis of the right femur.

FIG. 2. - At the age of 5 years the x-ray film shows shortening of the femur and gross deformity of the lower articular surface of the right femur. joints but in some cases there is surprisingly little deformity or disability following the joint infection. Any deformities which do occur are due to damage to the articular ends of the bones and not to adhesions in the joint. Generalized osteitis of the newborn is less common than the benign form and before the introduction of antibiotics the outlook was always grave. It is possible that some cases included in the 'benign' group would have progressed to the severe form if they had not been treated with antibiotics. Seven patients with the severe type of neonatal osteitis have been seen since 1945. All were gravely ill when first seen.

\section{Case Reports}

Case 1. A girl, well until 2 weeks old, was admitted with swelling of both thighs and the right knee. Blood culture grew coagulase-positive staphylococci. (The sensitivity of the organism to penicillin was not estimated.) Penicillin was given by intramuscular drip in a dose of 100,000 units daily for 11 days (totat $1 \cdot 1$ mega units). A soft tissue abscess of the right thigh was aspirated and pus was aspirated from the right hip and knee joints. Radiography revealed new subperiosteal bone over the shafts of both femora. Four weeks after the onset of the illness there was evidence of destruction of the right lower femoral epiphysis (Fig. 1). There was a subsequent interference with growth of the right leg, and five years after the onset of osteitis there was $3 \mathrm{~cm}$. shortening and gross deformity of the articular surfaces (Fig. 2). Movements of the knee joint are still free and painless.

Case 2. A boy developed a paronychial whitlow of the left thumb and right index finger six days after birth. Two days later there were red tender swellings of the left arm, right thigh and both legs. Arthritis developed in both knees and coagulase-positive, penicillin-sensitive staphylococci were grown from the aspirated fluid. Radiography showed that periosteum was raised over the left humerus and left radius and lesions in the metaphysis of the lower left femur and upper left tibia. Penicillin, $1 \cdot 5$ mega units, was given by intermittent intramuscular injection in eight days. Parotitis developed on the third day but none of the swellings was aspirated and the infant's condition ceased to give rise to anxiety after the fourth day of treatment. Recovery was. complete with resolution of all bone lesions and recovery of complete movement of both knee joints.

Case 3. A boy was admitted at the age of 3 weeks with a tentative diagnosis of infantile cortical hyperostosis. There was a firm red swelling over the left clavicle, swelling of both thighs and right leg and effusion in both knees. Radiography revealed osteitis of the left clavicle (Fig. 3), an area of rarefaction in the right ilium and periostitis of both femora with effusion in both knee joints. Penicillin was administered in a dosage of 20,000 units three-hourly by intramuscular injection. After two days the clavicular swelling became fluctuant and it was aspirated. The temperature continued to fluctuate. Culture of the pus revealed a coagulasepositive staphylococcus, resistant to penicillin but sensitive to streptomycin and aureomycin. On receipt of the report penicillin therapy was stopped and the infant was given $25 \mathrm{mg}$. aureomycin six-hourly. After 24 hours the temperature became normal and the child's condition improved rapidly. After seven days the hospital supply of aureomycin was exhausted and penicillin therapy was restarted with a dose of 50,000

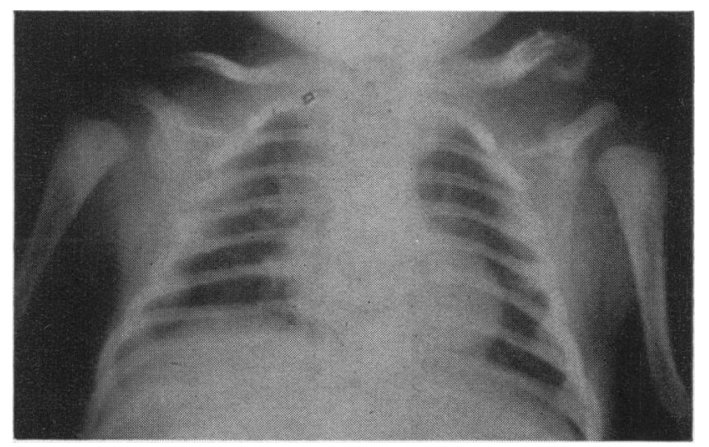

FIG. 3.-Osteitis of the left clavicle. 
units four-hourly. Within 24 hours the temperature was again elevated. The knees were aspirated but irregular pyrexia continued until aureomycin became available five days later. Two weeks after admission a large intrapelvic abscess was evacuated. Both knees

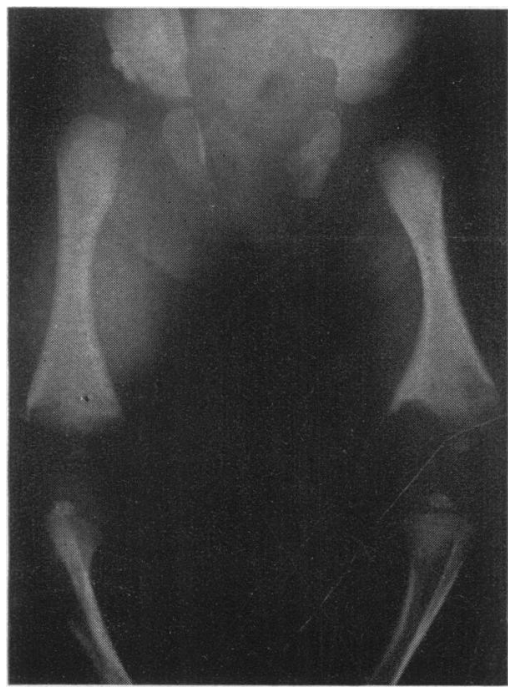

Fig. 4.-Radiography shows lesion in right ilium and gross arthritis of knees with bone destruction at lower end of both femora.

Fig. 5.-G ross destruction of lateral aspect of lower right femur and medial aspect of the lower left femur.

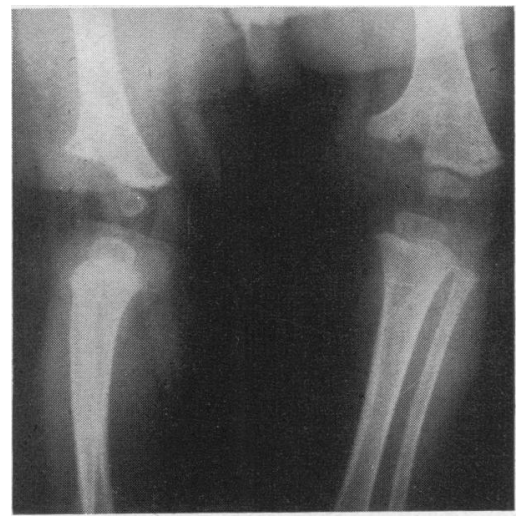

were aspirated on four occasions and all specimens revealed a penicillin-resistant staphylococcus. During the fourth week of treatment the temperature became normal and thereafter the patient's general condition improved rapidly. Four weeks after admission radiography revealed gross arthritis of both knees with bone destruction and partial subluxation (Fig. 4). The infant was discharged after 14 weeks. At the age of 8 months movement of both knees was full and free but radiography revealed gross damage to the ends of both femora (Fig. 5). It is still too early to give anything but a guarded prognosis on future bone growth and final joint function.

Case 4. A boy was well until 3 weeks of age when he developed diarrhoea. He was treated at home for

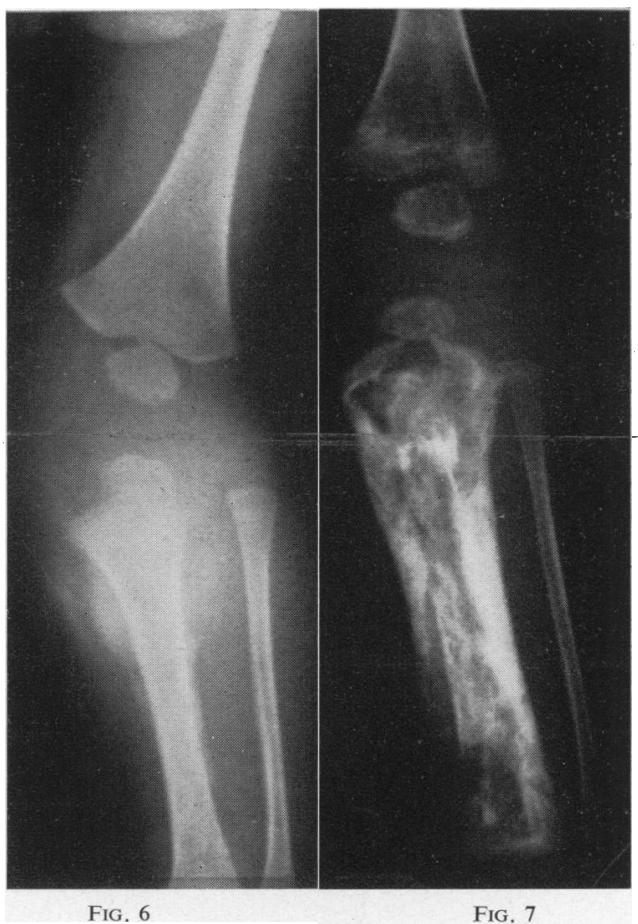

FIG. 6.-Extensive osteitis of tibia.

FIG. 7.-Extensive involucrum formation and gross destruction of the left tibia six months after onset.

a week with rectal chloromycetin. The diarrhoea improved but his general condition deteriorated and he developed a red swelling over the right shoulder. He was admitted to hospital with a generalized rash and all the features of septicaemia. There was gross swelling of the entire right leg and pus was aspirated from the right knee joint. Coagulase-positive staphylococci were grown from the blood, from the urine, from a rectal swab and from the knee joint fluid. Aureomycin, 100 mg., was given intravenously and thereafter orally in a dose of $25 \mathrm{mg}$. six-hourly. Radiography revealed oedema of the soft tissue of the leg but no bone lesion. The staphylococcus was reported sensitive to streptomycin and aureomycin but resistant to penicillin. Four days after admission the left knee was aspirated. The staphylococcus isolated from the fluid was reported sensitive to penicillin. Two days later a small subperiosteal abscess over the head of the tibia was evacuated and the wound sutured. The organism was reported sensitive to streptomycin and moderately sensitive to aureomycin. On the eighth day the general condition had improved and the temperature was normal. Aureomycin was discontinued. As the haemoglobin was only $65 \%$, a small blood transfusion was given. The child remained well until the thirteenth day when the temperature rose to $103^{\circ} \mathrm{F}$. and he once more became seriously ill. Signs of bronchopneumonia appeared and were confirmed by radiography. 


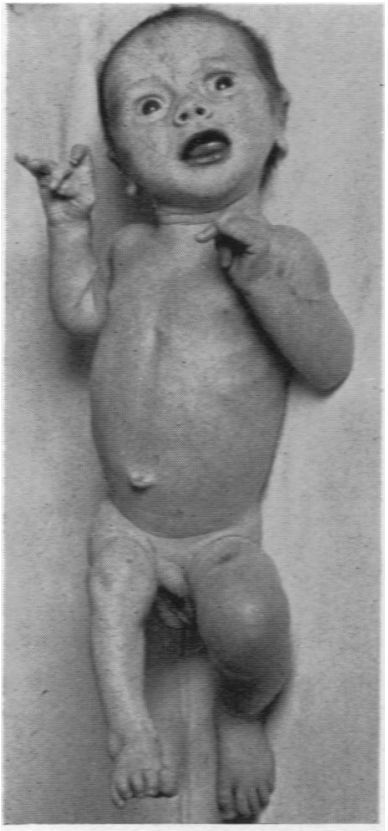

FIG. 8

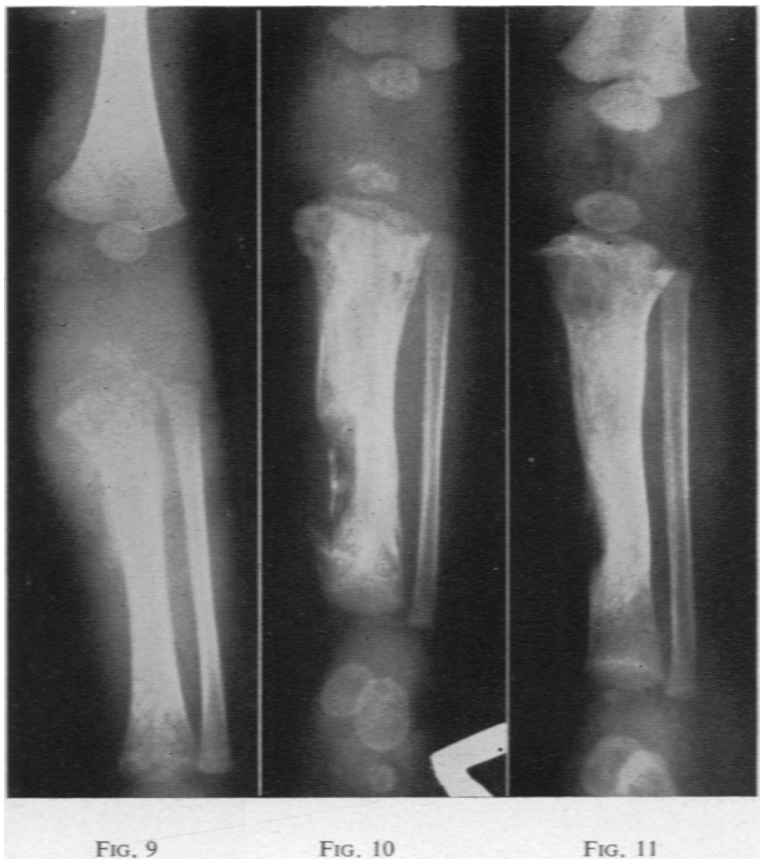

FiG. 8.-Photograph showing discoloration and swelling of left lower limb and right arm. Child toxic and marasmic.

FrG. 9.-Osteitis of left tibia.

Fig. 10.-Radiography shows free sequestrum two months after onset.

Fig. 11.-Radiographic appearances four weeks after sequestrectomy showing early consolidation of tibia.

Radiographs also revealed extensive osteitis of the tibia (Fig. 6). Aureomycin therapy was begun again in a dose of $50 \mathrm{mg}$. six-hourly. The general condition slowly improved and after the nineteenth day the temperature remained normal. Aureomycin was discontinued on the twenty-sixth day. The child was dismissed from hospital after three months. Six months after the onset of the illness radiography showed extensive involucrum formation and gross destruction of the left tibia (Fig. 7). Movement of the knee joint was full and painless.

The child was re-admitted three months later with persistent pyuria. Radiography revealed multiple calculi in the left kidney. Several stones were removed but kidney function remained poor on that side and eventually the kidney was removed. The pathologist reported chronic pyelonephritis with multiple calculi. The child made a rapid recovery from the nephrectomy but he will probably require sequestrectomy at a later date.

Case 5. A boy was well until 3 weeks old, when he developed a left inguinal abscess. This was incised but the pus was not examined. Three days later the left leg was red and swollen and on admission to hospital the following day the right arm was also red, hot and swollen (Fig. 8). He was marasmic and suffered from a toxic gastro-enteritis. Penicillin, 50,000 units, was injected intramuscularly and pus was aspirated from the right arm and left leg. Penicillin was continued in a dose of 25,000 units three-hourly. The organism proved to be a coagulase-positive staphylococcus, resistant to penicillin but sensitive to streptomycin and aureomycin.
On receipt of this report two days after admission. penicillin was discontinued and oral aureomycin was given in a dose of $50 \mathrm{mg}$. six-hourly. The infant was now gravely ill with deep jaundice and enlargement of the liver and spleen. Three days after admission pus was aspirated from a whitlow of the left index finger. After four days of aureomycin therapy, the general condition began to improve, vomiting and diarrhoea had ceased, and the infant showed more interest in his feeds. Radiography showed infiltration of both lung fields and soft tissue swelling of the right arm and left leg. Ten days after admission jaundice was fading but there was gross swelling of the left knee. Pus was aspirated from the knee joint and streptomycin instilled. Two days later there were pyaemic lesions of the fifth left finger, the right palm and the right wrist. Pus was aspirated from each site. Radiography at this time revealed osteitis of the left tibia (Fig. 9) and an area of

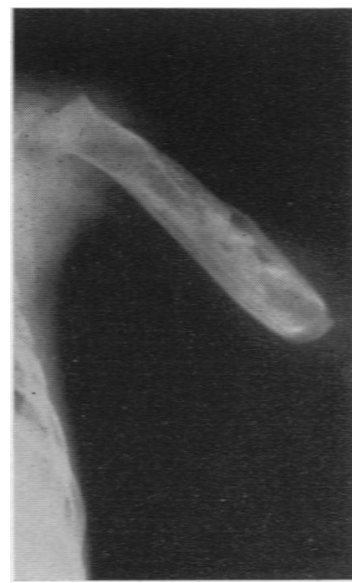

FrG. 12-Radiographic appearance of left humerus at age of 6 months. translucency in the neck of the right radius. Following our experience with Case 4 aureomycin was continued for four weeks. Radiography at this time revealed 
healing lesions of the right radius and proximal phalanx of the left index finger and extensive osteitis of the left tibia. Two months later a radiograph revealed a free sequestrum (Fig. 10). Sequestrectomy was performed and a subsequent radiograph showed the tibia consolidating (Fig. 11).

At the age of 5 months the child reported with a painless swelling over the left deltoid. The swelling was superficial and radiography revealed no bone lesion. Aspiration revealed a coagulase-positive staphylococcus, resistant to penicillin but sensitive to aureomycin. Radiography two weeks later showed extensive osteitis of the left humerus (Fig. 12). The general condition remained excellent but a month later he was re-admitted with a painless superficial swelling of the upper left thigh. Radiography revealed early osteitis of the left ilium. Aureomycin therapy was begun and the abscess was aspirated and $0.25 \mathrm{mg}$. of aureomycin in a glycine diluent was instilled. The coagulase-positive staphylococcus grown from the pus was reported resistant to penicillin but sensitive to aureomycin and chloromycetin. The child is still under treatment.

Case 6. A girl became cross and fevered, with loose stools, at the age of 3 weeks. (A week previously the umbilical cicatrix had been mildly infected.) The child became gravely ill and she was treated at home with oral chloromycetin. At 4 weeks of age the right leg became swollen and this swelling slowly subsided until it became localized over the right ilium two weeks later. Radiography revealed early osteitis of the right ilium. The general condition was not satisfactory: $8 \mathrm{ml}$. of thick pus was aspirated and $0 \cdot 5 \mathrm{~g}$. streptomycin instilled. The coagulase-positive staphylococcus grown from the pus was reported resistant to penicillin but sensitive to aureomycin and chloromycetin. Oral chloromycetin was discontinued after four weeks and radiography at this time revealed extensive osteitis of the ilium (Fig. 13). A week later the swelling over the right ilium recurred. The child was admitted to hospital and aureomycin therapy was begun. Marrow puncture revealed a penicillin-resistant staphylococcus, and $0 \cdot 1 \mathrm{~g}$. aureomycin in a glycine diluent was instilled into the marrow. Marrow puncture and instillation of aureomycin was repeated a week later. A scanty growth of a coagulasepositive staphylococcus was reported. The infant is

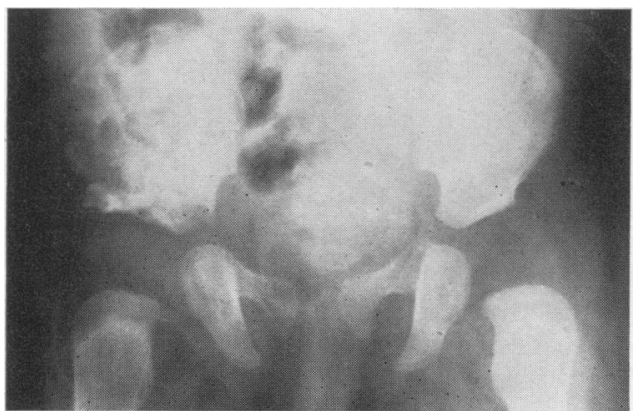

FIG. 13.-Radiographic appearance of the right ilium showing extensive osteitis at the age of 8 weeks. still under treatment. The general condition remains satisfactory but there is gross thickening of the ilium.

Case 7. At the age of 3 weeks this boy was admitted to a fever hospital suffering from gastro-enteritis. He was treated with 50,000 units of penicillin four-hourly and dextrose and plasma by intravenous drip. After two weeks an abscess appeared at the site of the cutdown in the right leg. Proteus was grown from the aspirated pus. A week later the left knee became swollen and aspiration revealed a coagulase-positive staphylococcus resistant to penicillin but sensitive to aureomycin. The infant was transferred to this hospital. On admission he had gross swelling of the left knee and fullness and limitation of movement of both hips. Aureomycin therapy was begun. Radiography revealed arthritis of the left knee and osteitis of the upper ends of both femora (Fig. 14). Both hip joints and the left knee joint were aspirated and $0.25 \mathrm{~g}$. streptomycin instilled. Marrow fluid was aspirated from both upper femoral metaphyses. The fluid from all three joints was reported sterile, but marrow fluid showed a coagulasepositive staphylococcus resistant to penicillin and aureomycin but sensitive to chloromycetin. The limbs were immobilized in abduction to prevent subluxation of the hips. Aureomycin was discontinued and chloromycetin substituted in a dose of $50 \mathrm{mg}$. six-hourly.

The child is still under treatment. Already it appears that irreversible damage has been done to the upper end of the left femur.

\section{Discussion}

Acute haematogenous osteitis occurs most commonly between the ages of 2 and 12 years and the causal organism is a coagulase-positive staphylococcus in $95 \%$ of cases (White and Dennison, 1952). In a series of 212 cases of acute osteitis treated in one surgical unit between 1936 and 1950 only seven infections were due to a streptococcus.

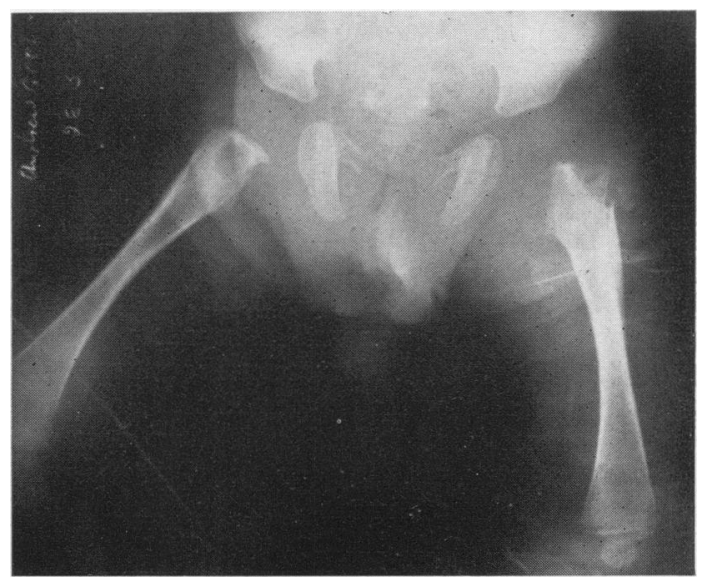

FIG. 14.-Radiograph shows osteitis of upper ends of both femora and arthritis of hips and subluxation of left hip. 
There were three pneumococcal infections and the remaining 202 cases were caused by a staphylococcus. While pyogenic bone infection is not uncommon in the first two years of life, the infecting organism is commonly a streptococcus. These infections are relatively benign and only rarely endanger either life or limb. Streptococcal osteitis of infancy should be classed as subacute osteitis (White and Dennison, 1947).

Osteitis of the newborn is almost invariably staphylococcal. Although it has already been stated that the disease presents in either a benign or a severe form, it may be impossible in the early stages to foretell which course the disease will take. Since 1946, 17 cases of staphylococcal osteitis of the newborn have been treated in the unit. In 10 the organism was resistant to penicillin. In 102 cases of acute staphylococcal osteitis in older children treated during the same period the organism was sensitive to penicillin in all but one case. Of the neonatal cases, 10 were benign, and although the staphylococcus was resistant to penicillin in five, the general condition of the patient never gave rise to anxiety and all made a full recovery. Seven cases of severe neonatal osteitis have been reported in some detail. The systemic infection was grave in all seven. In Case 2 the staphylococcus was sensitive to penicillin; in Cases 3 to 7 , it was penicillin-resistant. Sensitivity was not assessed in Case 1, but, from the gross bone damage which occurred in spite of penicillin therapy, it is possible that the staphylococcus in this case was penicillinresistant.

In Glasgow the incidence of acute haematogenous osteitis in childhood remained almost constant during the period 1930-1949 (White, 1937; Dennison, 1948). During the past 18 months, however, the disease has become exceedingly rare. Yet five cases of neonatal osteitis of the severe type have been treated in the unit during the past year. The causal organism in all five was a penicillin-resistant staphylococcus.

It is now our practice to assume that all neonatal staphylococcal infections are caused by a penicillinresistant organism and to administer aureomycin from the beginning of treatment. Should the organism be reported penicillin-sensitive, aureomycin can be stopped and treatment continued with the more readily available penicillin. Following our experiences with Cases 3 and 4, we now give aureomycin for a much longer period than is usually recommended. A minimum period of three weeks is considered essential in any case of generalized osteitis of the newborn. As with penicillin therapy, neither the general condition of the patient with osteitis nor the temperature chart are reliable criteria for cessation of therapy (Dennison, 1948). As penicillin is bacteriocidal and aureomycin and chloromycetin are bacteriostatic, it is probably even more dangerous to stop aureomycin and chloromycetin treatment too early. No ill effects have followed prolonged courses of aureomycin or chloromycetin. And now in Case 7 we are faced for the first time with the problem of dealing with an organism which has apparently become resistant to aureomycin.

Subperiosteal and soft tissue abscesses should be evacuated using a wide-bore needle. This must be done before increased tension leads to necrosis of the overlying skin. As in older children, joint infection is treated by aspiration followed by penicillin instillation. This procedure is repeated as required. When the organism is resistant to penicillin, streptomycin or aureomycin in a glycine diluent is instilled into the joint instead of penicillin.

While the percentage recovery to full function in cases of osteitis of childhood is gratifyingly high, the results in neonatal osteitis can be as bad as anything one experienced before the introduction of antibiotics. One can only presume that in the past such cases died of septicaemia before gross damage to bone and joint became apparent. Although the antibiotics are life-saving, irreversible damage is done to the bone in the early stages of the disease and the progress of the bone disease is influenced little by antibiotic therapy. There is gross deformity of the limb in Case 1; there will be serious deformity in Cases 3 and 7; the prognosis is still guarded in Cases 4, 5 and 6 . Case 2 should probably be included in the 'benign' group.

Summary

Osteitis in infancy differs from osteitis in older children.

The disease as it affects children under the age of 2 years falls into two groups: (1) streptococcal osteitis of infancy; (2) osteitis of the newborn. In both types joint infection is common but sequestrum formation is rare. Poorly timed surgical intervention may jeopardize a child's life.

Streptococcal osteitis of infancy is generally a mild disease.

Osteitis of the newborn appears in a benign and in a severe form. The infection is usually staphylococcal.

In 102 patients with acute osteitis over the age of 2 years, there has been only one penicillinresistant infection. In 17 cases of staphylococcal osteitis in the neonatal group, the organism has been resistant to penicillin in 10 cases. 
Seven cases of neonatal osteitis of the severe type are reported.

The treatment of staphylococcal arthritis by aspiration and instillation of penicillin gives satisfactory results when the organism is sensitive to penicillin. Streptomycin or the intravenous dosage of aureomycin may give equally good results when the organism is penicillin-resistant.

It is suggested that in the treatment of osteitis with aureomycin and chloromycetin the course should be longer than is usually recommended.

In the severe form of neonatal osteitis the diagnosis is rarely made before irreversible bone damage has taken place. Although the mortality is now low, the morbidity is still high.
The cases were all treated in Mr. Matthew White's wards and we thank him for his advice and encouragement. We also thank Dr. A. M. MacDonald and his staff in the pathology department for their constant help. The photographs and reproductions of $x$-ray films are the work of Mr. J. L. A. Evatt, A.R.P.S.

REFERENCES
Athanassiadis, C. N. (1951). J. Bone Jt Surg., 33в, 74.

Blanche, D. W. (1952). Ibid., 34A, 71.

Cass, J. M. (1940). Archives of Disease in Childhood, 15, 55. Compere, E. L., Schnute, W. J. and Cattell, L. M. (1945). Ann. Surg., 122, 954.

Dennison, W. M. (1948). J. Bone Jt Surg., 30B, 110

Dillehunt, R. B. (1935). Surg. Gynec. Obstet., 61, 96.

Fraser, J.' (1924). Brit. med. J., 2, 605.

Green, W. T. (1935). J. Amer. med. Ass., 105, 1835.

Greengard, J. (1946). Med. Clin. N. Amer., 30, 135.

Paschlau, G. (1933). Mschr. Kinderheilk., 55, 280.

Stone, S. (1942). Amer. J. Dis. Child., 64, 680

Thomson, J. and Lewis, I. C. (1950). Archives of Disease in Childhood, 25, 273.

White, M. (1937). Postgrad. med. J., 13, 381.

and Dennison, W. M. (1947). Brit. med. J., 1, 900. 